This document is the Accepted Manuscript version of a Published Work that appeared in final form in J. Chem. Phys. 135, 084501 (2011), copyright (c) American Institute of Physics after peer review and technical editing by the publisher. To access the final edited and published work see

jcp.aip.org 


\title{
Quantum-Classical Simulation of Electron Localization in Negatively Charged Methanol Clusters
}

\author{
Letif Mones ${ }^{\mathrm{a}}$, Peter J. Rossky ${ }^{\mathrm{b}}$ and László Turi ${ }^{\mathrm{a}, 1}$ \\ ${ }^{a}$ Eötvös Loránd University, Department of Physical Chemistry, Budapest 112, P. O. Box 32, \\ H-1518, Hungary \\ ${ }^{b}$ Department of Chemistry and Biochemistry and Institute for Computational Engineering and \\ Sciences, \\ University of Texas at Austin, Austin, TX 78712
}

A series of quantum molecular dynamics simulations have been performed to investigate the energetic, structural, dynamic and spectroscopic properties of methanol cluster anions, $\left[\left(\mathrm{CH}_{3} \mathrm{OH}\right)_{\mathrm{n}}\right]^{-},(n=50-500)$. Consistent with the inference from photo-electron imaging experiments, we find two main localization modes of the excess electron in equilibrated methanol clusters at $\sim 200 \mathrm{~K}$. The two different localization patterns have strikingly different physical properties, consistent with experimental observations, and are manifest in comparable cluster sizes to those observed. Smaller clusters $(n \leq 128)$ tend to localize the electron in very weakly bound, diffuse electronic states on the surface of the cluster, while in larger ones the electron is stabilized in solvent cavities, in compact interior-bound states. The interior states exhibit properties that largely resemble and smoothly extrapolate to those simulated for a solvated electron in bulk methanol. The surface electronic states of methanol cluster anions are significantly more weakly bound than the surface states of the anionic water clusters. The key source of the difference is the lack of stabilizing free hydroxyl groups on a relaxed methanol cluster surface. We also provide a mechanistic picture that illustrates the

\footnotetext{
${ }^{1}$ E-mail: turi@chem.elte.hu, fax: (36)-1-372-2592

molet@enzim.hu, fax: (36)-1-372-2592

rossky@mail.utexas.edu, fax: (1)-512-471-1624
} 
essential role of the interactions of the excess electron with the hydroxyl groups in the dynamic process of excess electron transition from surface-bound states to interior-bound states. 


\section{Introduction}

Solvated electrons in methanol have been investigated intensively in the last 50 years. ${ }^{1,2,3,4,5,6,7,8,9,10}$ The similarities and the differences of methanol as a solvent compared to water provide the main motivation for the scientific interest in studying electron solvation in methanol. A more complete understanding of electron solvation can be reached not only by varying the polar solvent but also by extending the investigations from bulk solvents to finite size solvated electron systems, negatively charged solvent clusters. These cluster anions represent a transitional state between the gas phase and condensed phase species, but the experimental signatures of solvation are more clearly observed and interpreted in clusters.

Methanol cluster anions of various sizes have been prepared and characterized by the Neumark group using photoelectron imaging technique., ${ }^{9,10}$ They observed two markedly different sequences with cluster size in the excess electron vertical detachment energy (VDE) suggesting the presence of two different cluster isomer classes with distinct binding motifs. Of the two patterns, the more strongly bound features (type I) were assigned to structures that internally solvate the excess electrons (interior-bound excess electron states) in analogy to the bulk solvated electron. The significantly more weakly bound states (type II), on the other hand, were assumed to indicate the presence of surface-bound excess electrons. Most recently, the dynamics of electron solvation in iodide-doped methanol clusters following photoexcitation were studied by the same group. ${ }^{11}$

In comparison, the VDE of water cluster anions ${ }^{12,13,14,15}$ has a more complex size dependence with at least three distinct binding motifs. Although the precise classification for water cluster anions is still to be resolved, it appears that in addition to the position of the excess electron within the cluster, ${ }^{15}$ the conditions of the cluster preparation also strongly influence the experimental signals. ${ }^{13,16}$ Analogous observations were reported for methanol cluster anions. At low backing pressure, producing warmer, and therefore more likely 
structurally relaxed methanol clusters, Ref. 9 reports that the putative interior-bound state appears only for $n \sim 140$ and larger, while the putative surface-bound state is not at all evident for $n>190$. Thus, one could infer that for relaxed "warm" clusters, the surface-dominant to interior-dominant transition with increasing $n$ occurs in the approximate range $140<n<190$. At high backing pressure, where cold and metastable clusters are hypothesized to occur, ${ }^{13,16}$ the interior-bound state persists for $n>140$, but the relatively weakly bound putative surface state becomes prominent for all $n>70$. The aim of the present paper is to provide a more detailed structural identification for the two classes of methanol cluster anions using molecular modeling techniques. This approach should also provide additional molecular level information about the energetics, dynamics and spectroscopy of cluster anions.

Here, we address the problem using mixed quantum-classical molecular dynamics (QCMD) simulations. QCMD simulations that treat the excess electron quantum mechanically and the solvent bath classically, offer a reasonable alternative ${ }^{17,18,19}$ to the more demanding fully quantum calculations. In fact, all-electron quantum calculations are still limited to relatively small system size (i.e. few tens of atoms). The only study that investigated small methanol cluster anions (up to six monomers) using density functional theory methods with modest basis sets were published some time ago. ${ }^{20}$ This study indicated that both the interior excess electron states and the diffuse surface electron states are likely to represent minima on the potential energy surface in small clusters $(n=2-6)$. In contrast to the quantum chemistry calculations, the QCMD approach provides a theoretical framework for studying the electron solvation problem in systems containing up to several thousand atoms. The physical properties of a bulk solvated electron in methanol were characterized in a series of QCMD based studies, ${ }^{21,22,23,24,25,26,27}$ in good general agreement with experimental observations. Nevertheless, inaccuracies in the simulated absorption spectrum of the solvated electron in methanol $^{22}$ motivated the improvement of the electron-methanol molecule interaction 
potential for further studies. Accordingly, a new electron-methanol molecule pseudopotential using static exchange pseudopotential theory was developed and applied in QCMD simulations recently. ${ }^{28}$ These simulations notably improved the position and the half-width of the optical spectrum of the solvated electron in bulk methanol in comparison with experiments. $^{3}$ This is important since, for the clusters experimentally assigned as having interior-bound electrons a strong detachment feature of the two-photon photoelectron spectrum using $1.55 \mathrm{eV}$ excitation indicated the presence of a well-defined excited state that was accessible at $1.55 \mathrm{eV}{ }^{9}$ This would be consistent with the existence of a cluster-supported excited state similar to the broad absorption evident for the bulk solvated electron in methanol, peaked experimentally at $1.9 \mathrm{eV} .^{3}$ The structural properties computed using the new pseudopotential are also in excellent agreement with those inferred from the electron spin echo measurements of Kevan. ${ }^{2}$ In a subsequent step, we characterized the excess electron localization on neutral equilibrated methanol clusters. ${ }^{29}$ The investigated scenario, adding an electron to neutral clusters, may represent the simplest model of cluster anion preparation by mimicking low energy electron attachment to pre-existing equilibrium methanol clusters. We found, that similarly to water clusters,${ }^{30}$ the pre-existing instantaneous dipole moment of the neutral clusters can bind the excess electron. Since we find that the surface of the neutral cluster is covered predominantly by the non-polar methyl groups, as suggested by earlier experiments, ${ }^{31}$ the initial localization takes place in weakly bound surface states. Excess electron attachment to stable interior bound states was not observed in either finite size methanol clusters or in the structures characterizing the equilibrium bulk (unrelaxed) solvent.

As the last step of this series of investigations, here we report the results of the simulation of excess electron and cluster relaxation, we follow the dynamics and characterize the fully relaxed states of methanol cluster anions. Comparison of the computed results to available experimental data will also be given. We note here that the simulations usually 
correspond to well-defined, i.e. thermal equilibrium, conditions and cluster experiments are expected to be non-thermal. ${ }^{32,33}$ Nevertheless, simulations can still provide important information on key energetic and structural aspects of cluster anions. This has been illustrated in previous simulation papers for water cluster anions, ${ }^{15,16,34}$ and we present here another example, for negatively charged methanol clusters.

\section{Methods}

To investigate the physical properties of relaxed methanol cluster anions, we performed mixed quantum-classical molecular dynamics simulations of 11 different cluster sizes in the range from $n=50-500$ solvent molecules equidistantly spaced on the $n^{-1 / 3}$ scale. The interaction between the classical methanol molecules was described by the 6-site OPLS (Optimized Parameters for Liquid Simulations) ${ }^{35}$ force field, while the excess electron responds to the methanol molecules via the recently developed pseudopotential. ${ }^{28}$ The electron-methanol pseudopotential was derived using a similar procedure to that implemented for a previous electron-water molecule pseudopotential. ${ }^{36,37}$ Mixed QCMD simulations on an excess electron bound to neutral water clusters, ${ }^{30}$ relaxed anionic water clusters,,${ }^{154}$ electron

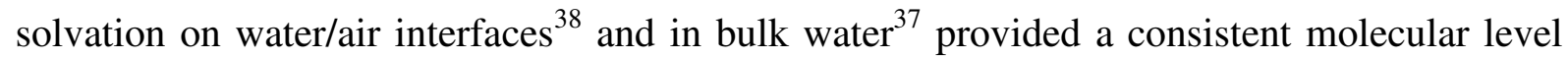
picture of the electron hydration phenomenon. Although, due to the inherent approximations of the pseudopotential, one may not expect that these simulations will predict physical properties (e.g. binding energies) quantitatively, the findings for water cluster anion systems suggest that the pseudopotential-based method can be used with confidence in a wide range of stabilization energies for semi-quantitative purposes, and that is the goal in the present work. The results of most recent model-free $a b$ initio molecular dynamics simulations on small water cluster anions also support this claim. ${ }^{39,40}$ 
The wave function of the excess electron was represented in a plane-wave basis ${ }^{18}$ on $32 \times 32 \times 32$ discrete grid points equidistantly distributed in a box of $25 \AA$ length. ${ }^{41}$ Previous study of localization of an excess electron on equilibrated neutral methanol clusters showed that the electron initially occupies a very diffuse surface bound orbital. ${ }^{29}$ The excess electronic states are classified by geometric parameters, the radius of the cluster $\left(r_{c}\right)$, the radius of gyration of the electron $\left(r_{e}\right)$ and the distance between the centers of mass of the cluster and the electron $(R)$. Interior bound states are identified by the inequality $R+r_{e}<r_{c}$, while for surface states $R \geq r_{c}$.

In the present work we considered two relaxation scenarios depending on the initial conditions of the molecular dynamics trajectories. This procedure has been used previously to analyze electron solvation dynamics in water clusters. ${ }^{34}$ To separately analyze surface state dynamics and interior state dynamics, simulations were launched from either pre-formed surface states or pre-formed interior states. We used pre-equilibrated neutral methanol clusters at $T=200 \mathrm{~K}$ as initial configurations to investigate the dynamics of surface-bound electron methanol clusters. The equilibration process of the neutral clusters was the same as in Ref. 29. We introduced the excess electron to the last configuration of the equilibrium trajectory of the neutral clusters and followed the subsequent trajectory. ${ }^{42}$ For the interior states, simulations were initiated from configurations with the electron bound in a solvent cavity, in the centre of the clusters. These systems were initially created from previous equilibrium bulk simulations with 1600 methanol molecules by simply cutting out a system with the desired number of molecules nearest to the electron center-of-mass.

The Schrodinger equation of the excess electron was solved by an iterative and block Lanczos procedure. ${ }^{18}$ The quantum forces were evaluated using the Hellmann-Feynman theorem. ${ }^{18}$ The time step of the simulations was $1.0 \mathrm{fs}$ and we used the velocity Verlet algorithm to integrate the equations of motion. ${ }^{43}$ Total simulation times for each trajectory 
were at least $250 \mathrm{ps}$, and most were longer, as will be evident. We also computed the optical absorption spectrum of the cluster anions similarly to that for the bulk solvated electron in methanol, taking only the first seven transitions into account. ${ }^{28}$ To compare the cluster simulations to bulk results, we performed a solvated electron simulation in bulk methanol at $T=200 \mathrm{~K}$ with $n=200$ molecules, following that reported previously for $T=300 \mathrm{~K}^{28}$ All cluster simulations were carried out in the microcanonical ensemble.

\section{Results}

We start the discussion with the pre-formed surface excess electronic states. Figure 1 shows the time evolution of the ground state energies for these trajectories. Clearly, in all these examples, the clusters bind the electron very weakly at the outset. Geometry analysis indicates that the excess electron occupies a weakly stabilized state on the surface in all clusters in the first $25-50$ ps. The radius of gyration reflects a highly diffuse electron distribution with a nominal radius of $8-10 \AA$ (Figure 2). The electron in this diffuse state is bound by the instantaneous dipole moment of the cluster. Based on the behavior of the excess electron after $\sim 50$ ps of the relaxation, three different types of clusters can be identified. In the smallest clusters $(n=50-85)$, the electron stays bound on the surface during the timeframe of the simulation $(\sim 250 \mathrm{ps})$. The excess electronic states remain weakly stabilized and very diffuse in these clusters. As the cluster size increases, a new trend appears at around $n \sim 100$, indicating the appearance of a different type of electron binding motif with the electron entering into the interior of the cluster. With the penetration, the radius of the electron gradually shrinks to $\sim 2 \AA$, a value characteristic of the bulk solvated electron in methanol. ${ }^{2,28}$ In the case of middle size clusters (103-161 molecules), the electron is able to enter temporarily into the interior, but the electron then returns to the surface (see in Figures 1 and 2). This suggests a bistable size regime. For larger clusters (205-500 molecules) the electron 
penetrates into and remains in the interior, as can be seen from the ground state energy and the compact radius of gyration in Figures 1 and 2 . From the limited study here, the time required for the electron to form stable interior state does not necessarily correlate with the size of the cluster, but a statistical set of trajectories would be needed to analyze this aspect in detail. Nevertheless, these surface-initiated trajectories indicate that at $T \approx 200 \mathrm{~K}$, the relaxation of the cluster takes several hundred picoseconds. We will analyze the dynamics in more detail below.

To gain additional insight into the electron relaxation in methanol clusters, we generated trajectories from pre-formed interior states (see Sec. II). We note that while the surface initiated trajectories can be considered as simplified models for the electron attachment of a low-energy electron to neutral clusters, the trajectories launched from interior states have no physical counterpart, but can provide complementary information. Accordingly, the general trends of the interior launched simulations mirror those of the surface states as reflected in the time evolution of the ground state energies (Figure 3) and radii of gyration (Figure 4). All trajectories start with deep ground state energy and a compact electron distribution. For the smallest clusters (including 50-103 molecules) the electron does not remain inside the clusters but it migrates to the surface. During this process, the electron binding energy gradually decreases (Figure 3), while the radius increases (Figure 4). Not surprisingly, the time required for the electron to reach the surface increases with the cluster size. For the largest simulated clusters (containing 128-500 molecules) we observe that the electron remains in the interior state during the timeframe of the simulation. These data on relaxation taken together indicate an exterior-interior transition at $200 \mathrm{~K}$ that occurs in the approximate size range $100<n<130$, very similar to the range reported from experiment of $140<n<190$, considering the uncertainties in the theoretical model and in the experimental cluster conditions. 
Evidently, the VDE (approximated here by the magnitude of the ground state energy) and the radius of the electron are characteristic properties of the electron binding motif of methanol cluster anions. Interior states are characterized by large VDE and compact electron distribution, while surface states have significantly weaker VDE and larger electron radius. This distinction is far stronger here than observed for water clusters. ${ }^{15,34}$ Figure 5 illustrates the correlation of these quantities showing the electron radius as the function of the VDE for interior initiated trajectories. In general, we conclude that the more compact the electron radius the more negative the ground state energy is. Clearly, the VDE's are clustered between 2.0 and $3.5 \mathrm{eV}$ with radii of 2.0-2.5 $\AA$ for interior states, while the surface state VDE falls most frequently between 0.0 and $1.0 \mathrm{eV}$ with $6-12 \AA$ radii. For interior states alone, the radius exhibits only a very narrow range.

We now turn to the relaxation of the trajectories. For the surface initiated clusters, significant relaxation in the ground state energies is not evident on the plotted time and energy scales of the trajectories while the electron is on the surface. Nevertheless, observable surface state relaxation becomes apparent in Figure 6. The binding energies of the surface states, averaged only over those configurations of the trajectories where the electron resides on the surface, are shown in Figure 6 (represented by triangles). That these energies are relaxed can be seen by directly comparing to those found previously in the static analysis (electron binding to unrelaxed neutral equilibrated clusters) ${ }^{29}$ (see squares and circles in Fig. 6). We note that, although the size dependence of the relaxed binding energy has the same trend as the static case, the average ground state energies become significantly lower (and the associated electron radii are smaller) during the dynamics. Figure 6 shows that the average ground state energies calculated for the dynamically relaxed surface state configurations are even much lower than those of the most stable $1 \%$ of the initial configurations of the static analysis (see circles in Fig. 6). Clearly, the electron is stabilized by structural relaxation at the 
surface of the clusters. Two factors play role here. We found that in the first 25-50 ps time interval of the simulation the electron need not remain in the vicinity of its initial localization site, but its center-of-mass visits a large portion of the cluster surface via the fast response of the diffuse, weakly bound electron to the nuclear motion. This effect makes it possible for the electron to find more favorable localization sites during the dynamics. Although slower, the solvent also responds by relaxing around the localizing charge by reorientation. We note that the overall relaxation of the surface state electron energy in methanol is significantly smaller than in water cluster anions. ${ }^{34}$ This difference can be explained by the very different surface character of water and methanol clusters. While the surface of methanol clusters is covered almost exclusively by apolar methyl groups, dangling hydrogen atoms of the free hydroxyl groups are abundant on water surfaces. The dangling hydrogens make the initial electron localization on water cluster surfaces more favorable and provide considerable flexibility for further relaxation.

Figure 6 also shows the average energies during the surface state segments of those trajectories that end up in an interior state. These values (denoted by open triangles on the figure) show a smoothly increasing VDE with size. The fact that the excess electron attached to larger clusters can survive for a significant amount of time on the apolar surface suggests that the penetration of the electron to the interior is, to some extent, kinetically hindered. The kinetic effect should depend on the local hydrogen-bonding structure in the vicinity of the electron localization site, and that is consistent with the observation that the penetration time of the electron to the interior is not simply a function of the cluster size in any given trajectory. We also observe that once the electron has entered the cluster, further relaxation, most likely attributable to the restructuring of the hydrogen-bonding network, occurs on a $\sim 100$ ps timescale, best reflected in the radius of the electron (Figure 2). Similar timescales can be recognized in interior-state initiated trajectories (Figure 4) that eventually end up in 
surface states. Solvent relaxation taking place in the trajectories where the electron remains in the interior is relatively minor, since these configurations already accommodate electronic states in solvent cavities with properly oriented methanol molecules.

Next, we explore the mechanism of penetration of an excess electron into the interior of a cluster. The general route is illustrated in Figure 7 for the $n=205$ cluster. A diffuse state of the excess electron (with an average radius of gyration, $\left\langle r_{e}\right\rangle=6.5 \AA$ ) prevails until the electron encounters a free hydroxyl hydrogen atom pointing out from the surface $(t \sim 108 \mathrm{ps})$. Although the hydrogen-electron center-of-mass distance is relatively large, $\sim 4.2 \AA$, the hydroxyl hydrogen provides extra stabilization for the electron, and this configuration apparently initiates the penetration. After another $\sim 4 \mathrm{ps,} \mathrm{due} \mathrm{to} \mathrm{the} \mathrm{local} \mathrm{rearrangement} \mathrm{of} \mathrm{the}$ nuclear environment, the electron approaches the hydrogen more closely ( $2.8 \AA$ ). At the same time, the excess electron becomes more compact with $r_{e}=4.2 \AA$. After an additional $\sim 10 \mathrm{ps,} \mathrm{a}$ nearby hydrogen-bond breaks, and a second hydroxyl hydrogen becomes coordinated to the electron. As can be seen well in the figure, in the next $~ 50$ ps the electron immerses gradually deeper into the cluster creating interactions with more hydroxyl hydrogen atoms (increasing coordination number) and, at the same time, its radius and its ground state energy decrease monotonically. During the process, a gradually deepening cavity is formed in the cluster surface as the electron enters into the cluster (131-153 ps). When the electron is deep enough inside the cluster, the formation of the outside solvent surface begins (153-173 ps). At this point, the electron can be considered as completely immersed in the cluster and occupies an interior state; after $173 \mathrm{ps,}$ neither the binding energy nor the radius of the electron change systematically. We note here that a more systematic analysis of the electron penetration dynamics using a statistical set of trajectories would be of interest as a separate study.

After examining the relaxation process, let us now turn our attention to the properties of the fully relaxed surface-bound and interior-bound excess electronic states. In the following 
analysis, we consider only relaxed portions of the trajectories where the electron persists for a minimum of $\sim 50 \mathrm{ps}$ in a surface state, ${ }^{44}$ or $\sim 250 \mathrm{ps}$ in an interior state. The vertical detachment energies averaged over these trajectory portions are collected in Figure 8 . The VDE values decrease approximately linearly with the inverse cube of the cluster size $\left(n^{-1 / 3}\right)$ for both the surface and the interior states in good agreement with the prediction of a continuum dielectric theory. ${ }^{45}$ This behavior was also found previously for water cluster anions. ${ }^{34}$ Figure 8 shows that although the simulated data somewhat overestimate the experimental VDE's, ${ }^{9}$ the simulated trends are remarkably similar to those of the experiments. Experimental type I and type II methanol clusters correlate nicely with the simulated interior state and surface state clusters, respectively.

It is generally expected that the linear extrapolation of the interior-bound and surfacebound binding energies to infinite size $\left(n^{-1 / 3}=0\right)$ results in the binding energy of the solvated electron in bulk methanol and on the methanol/air interface, respectively, at the same temperature. Linear extrapolation yields $\sim 3.5 \mathrm{eV}$ for the interior-bound states and $\sim 1.1 \mathrm{eV}$ for the surface-bound states. The former is higher by $\sim 1.1 \mathrm{eV}$ than the value calculated in the bulk simulations here at $200 \mathrm{~K}(2.4 \mathrm{eV})$. The main part of this difference is due to the neglect of the long-range interactions in the bulk simulations, similar to the observations for the aqueous case. ${ }^{34,37}$ We also note that simulation predicts a slightly more stable ground state for the bulk solvated electron at $200 \mathrm{~K}$ than at $300 \mathrm{~K}(2.1 \mathrm{eV}) .{ }^{28}$ The extrapolated interior state value of $3.5 \mathrm{eV}$ is in reasonably good agreement with the experimentally determined vertical electron binding energy of a bulk solvated electron in methanol, $3.10 \mathrm{eV}$ at $230 \mathrm{~K} .^{7}$ Figure 8 also contains the fitted linear extrapolation reported for the experimental cluster data. ${ }^{9}$ The data extrapolated to the bulk, predict $\sim 2.5 \mathrm{eV}$ and $\sim 0.7 \mathrm{eV}$ for type I and type II methanol cluster anions, respectively. Although the simulated values here are higher than those reported from cluster experiments, the simulated surface state data agree sensibly with type II 
methanol clusters. We also note, that while interior state simulations predict higher VDE's than these cluster experiments, the cluster experiment extrapolation gives a somewhat lower value than that of the bulk measurement. ${ }^{7}$ These differences likely lie both in the uncertain temperature of the cluster experiment and the approximate model used in the calculations. Nevertheless, the experiments and simulations predict very similar tendencies, the interior state (type I) clusters having significantly larger VDE than the surface state (type II) clusters.

We have also examined the optical spectrum of methanol cluster anions. Since the surface state clusters are very weakly bound, the excited states for these are unlikely to be well described by our model. Hence, we computed the spectra for the interior state cluster anions only. These spectra can be seen in Figure 9 for different size clusters. The position of the spectra does not show significant size dependence; all spectra are centered at $\sim 2.2-2.3 \mathrm{eV}$. The observation of this broad bulk-like absorption spectrum corresponds well to the indication of a similar absorption present in the clusters assigned experimentally as having interior-bound excess electron states. ${ }^{9}$ The $200 \mathrm{~K}$ peak location of the clusters is approximately $0.2 \mathrm{eV}$ blue-shifted relative to the $300 \mathrm{~K}$ bulk simulation ${ }^{28}$ reflecting a slight temperature dependence of the spectrum. The shape of the absorption bands lacks intensity at larger energies $(\sim 3-4 \mathrm{eV})$, similarly to the simulated bulk spectrum, which is undoubtedly due to the limitation of the electron-methanol molecule pseudopotential. ${ }^{28,46}$

\section{Discussion and Conclusions}

We have performed mixed quantum-classical molecular dynamics simulations to investigate the adiabatic relaxation of negatively charged methanol clusters following electron attachment to neutral clusters. We have shown previously that low energy electrons most likely attach to the surface of neutral clusters at the outset. ${ }^{29}$ The subsequent relaxation depends on various factors including the conditions of cluster preparation, the temperature, 
and the size of the clusters. As a simplifying assumption, the anion trajectories were started from equilibrated neutral nuclear configurations at $200 \mathrm{~K}$. The relaxation trajectories, depending on the size of the cluster, lead to two types of methanol cluster anion isomers. Smaller clusters $(n \leq 85)$ stabilize the excess electron in weakly bound, highly diffuse, surfacebound state, while for larger size clusters $(205 \leq n)$ the excess electron diffuses into the interior on a sub-nanosecond timescale. The trajectories launched from pre-formed interior state clusters result in stable final structures fully consistent with those obtained from pre-formed surface-bound clusters, with the same relaxation timescale for both types of trajectories.

We also explored the molecular process of electron penetration into the interior of the cluster and found that the initiating step is the formation of a stabilizing excess electrondangling hydroxyl interaction at the apolar methyl group-covered cluster surface. As other $\mathrm{OH}$ groups gradually exchange the local hydrogen-bonding with the solvent for coordination to the electron, the electron's energy deepens, its radius shrinks, and the electron is slowly drawn into the interior of the cluster.

Two types of relaxed cluster isomers, interior-bound and surface-bound anions, can be identified in complete support of the experimental interpretation. ${ }^{9}$ The analysis of VDE data as a function of the cluster size shows remarkable qualitative similarity to the experimental results. Since the computational one-electron pseudopotential model ${ }^{28}$ is very simple, it is not expected to reproduce the experimental data precisely. Nevertheless, not only do the simulations reasonably track the experimental VDE behavior for the two binding motifs, but the size range over which the most stable form transitions from surface to interior-bound are found to be comparable. Furthermore, a bulk-like optical absorption feature for interior-bound clusters is observed in simulation, as is indicated by experimental multiphoton photoelectron spectra. $^{9}$ 
In contrast to the case of water clusters, where there still remain unresolved questions about binding motifs, the emerging picture of electron solvation in methanol clusters appears to be significantly simpler, despite uncertainties in the model and in the experimental conditions. The main reason of the less complicated binding characteristics of the electron in methanol clusters is most likely due to the simpler surface topology of the methanol clusters. Unlike water clusters, where free $\mathrm{OH}$ groups provide possible strong electron binding sites on the surface, the methanol cluster surfaces are covered almost exclusively by non-polar methyl groups. With the methyl groups on the outside, the methanol cluster anions have a more limited set of low free energy structures leading to a simpler cluster energy landscape than in the aqueous case.

\section{Acknowledgements}

The work was supported by a grant to L. T. from the National Research Fund of Hungary (OTKA) under Contracts No. K75285. P. J. R. is grateful to the National Science Foundation (CHE-0910499) and the R. A. Welch Foundation (F-0019) for support of this work. 


\section{Figure Captions}

Figure 1. Time evolution of the excess electron ground state energy for smaller (upper figure: 50-black, 59-red, 85-blue, 103-magenta) and larger (lower figure: 128-black, 161-red, 205green, 269-blue, 361-magenta, 500-cyan) cluster anions launched from pre-formed surface states at $T \approx 200 \mathrm{~K}$.

Figure 2. Radius of gyration of the excess electron during the simulations for smaller (upper figure: 50-black, 59-red, 85-blue, 103-magenta) and larger (lower figure: 128-black, 161-red, 205-green, 269-blue, 361-magenta, 500-cyan) cluster anions launched from pre-formed surface states at $T \approx 200 \mathrm{~K}$.

Figure 3. Time evolution of the excess electron ground state energy for smaller (upper figure: 50-black, 59-red, 70- green, 85-blue, 103-magenta) and larger (lower figure: 128black, 161-red, 205-green, 269-blue, 361-magenta, 500-cyan) cluster anions launched from pre-formed interior states at $T \approx 200 \mathrm{~K}$.

Figure 4. Radius of gyration of the excess electron during the simulations of smaller (upper figure: 50-black, 59-red, 70- green, 85-blue, 103-magenta) and larger (lower figure: 128black, 161-red, 205-green, 269-blue, 361-magenta, 500-cyan) cluster anions launched from pre-formed interior states at $T \approx 200 \mathrm{~K}$.

Figure 5. Correlation between the ground state energy and the radius of an excess electron, from all simulated methanol clusters, initiated from pre-formed interior states at $T \approx 200 \mathrm{~K}$.

Figure 6. Average ground state energies for relaxed surface-bound excess electrons computed from pre-formed surface initiated trajectories (triangles) at $T \approx 200 \mathrm{~K}$. Trajectories that remain in surface states during the simulations are shown by full symbols, those that end up in interior states by open symbols. For comparison the average energies of an excess electron attached to equilibrated neutral (unrelaxed) cluster configurations (squares), and of the most stable $1 \%$ of these neutral configurations (circles) are also shown. ${ }^{29}$ 
Figure 7. Illustration of the mechanism of electron penetration into the cluster interior for $n=$ 205 methanol molecules at $200 \mathrm{~K}$. The electronic isosurface shown covers $80 \%$ of the excess electron density. The simulation time associated with each configuration is shown, along with the radius of gyration $\left(r_{e}\right)$ and the electron-solvent coordination number $\left(N_{c}\right)$.

Figure 8. Calculated (dashed lines) and experimental (solid lines) linear extrapolation of the binding energies of interior-bound (circle) and surface-bound (square) excess electron states at $200 \mathrm{~K}$. The calculated (hatched blue circle) $(T=200 \mathrm{~K})$ and experimental ${ }^{7}$ (solid blue circle) $(T=230 \mathrm{~K})$ binding energies in bulk are also shown. The cluster experiment extrapolation data are taken from Ref. 9.

Figure 9. Optical absorption spectrum calculated for interior state excess electrons in methanol clusters (161-red, 205-green, 269-blue, 361-magenta, 500-cyan) at a nominal temperature of $200 \mathrm{~K}$. 
Figure 1. Mones, Rossky and Turi

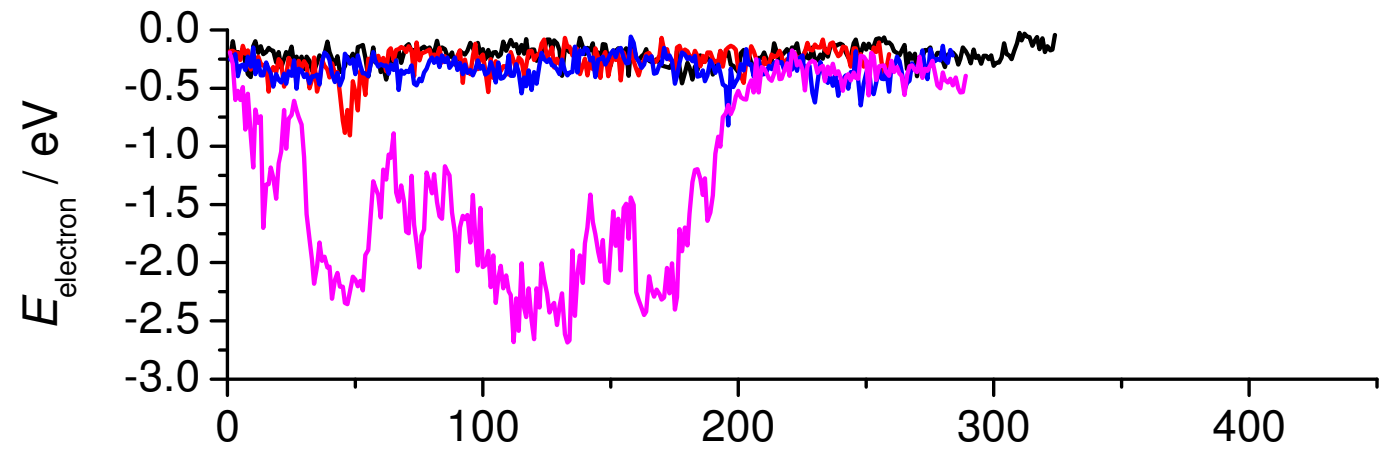

$t / \mathrm{ps}$

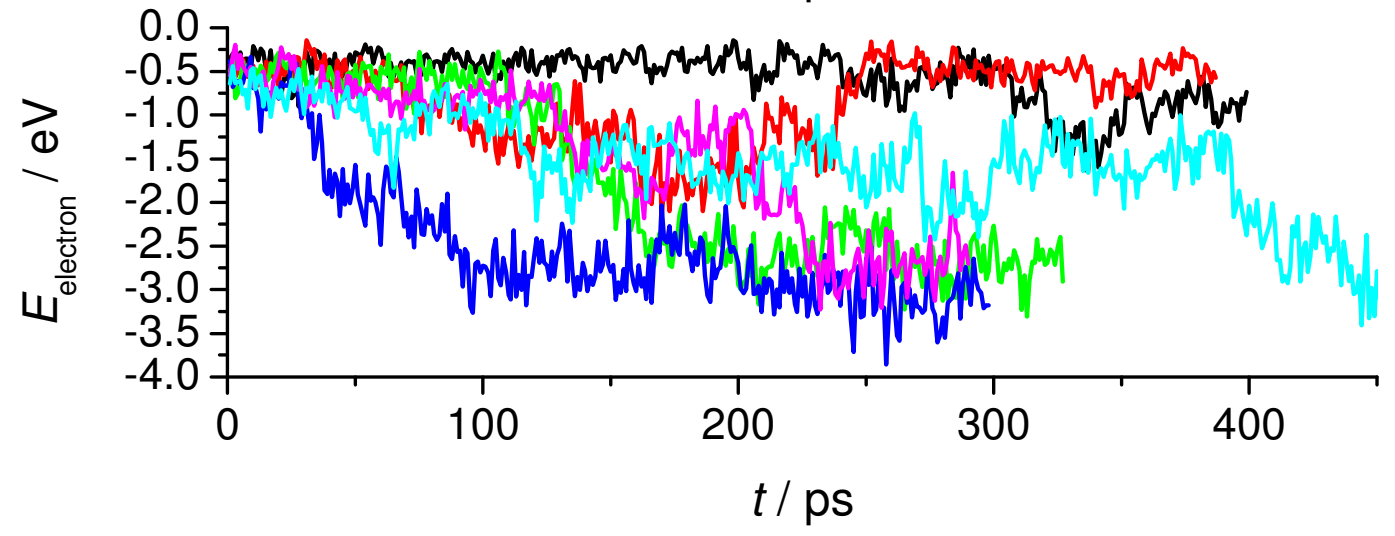


Figure 2. Mones, Rossky and Turi
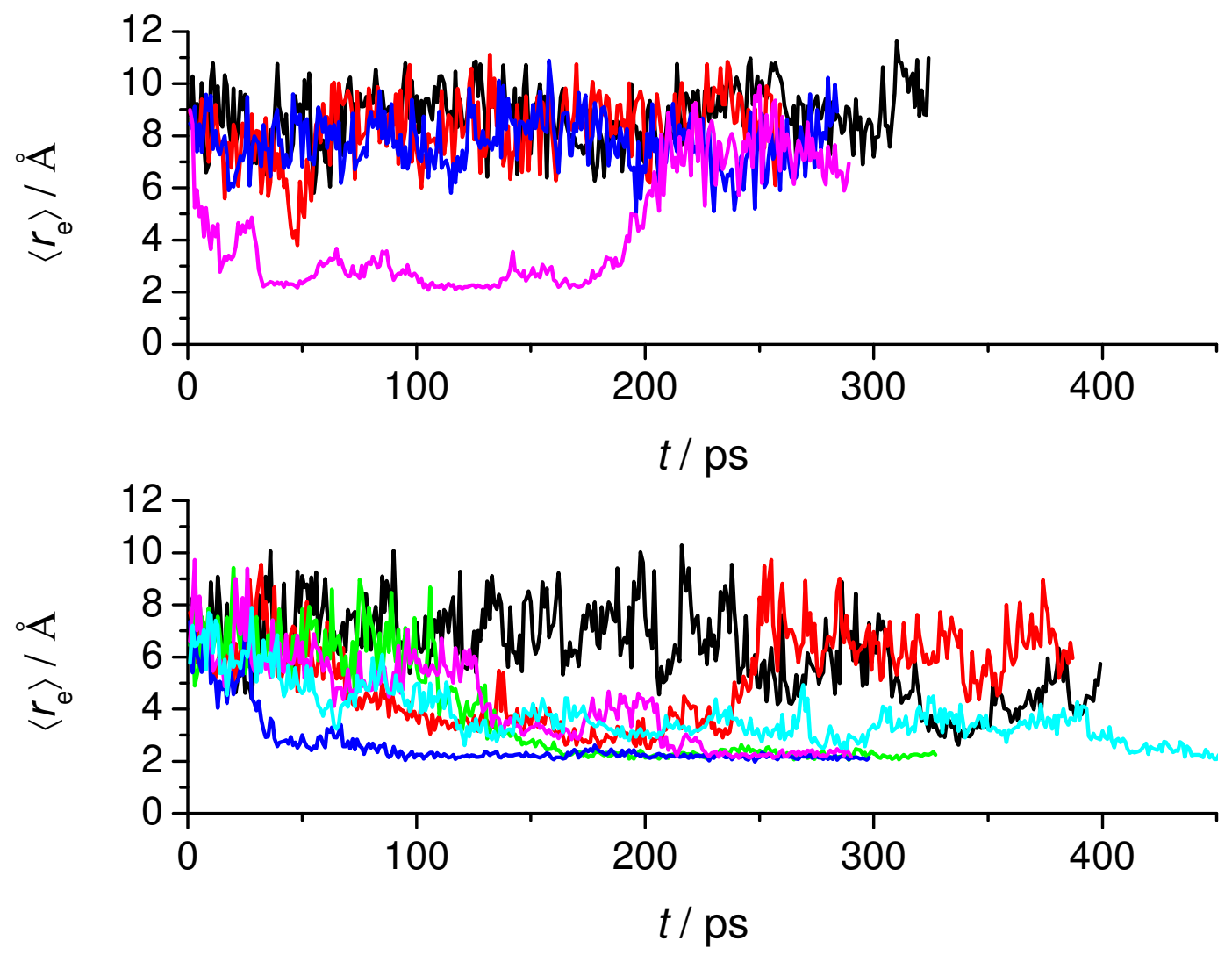
Figure 3. Mones, Rossky and Turi
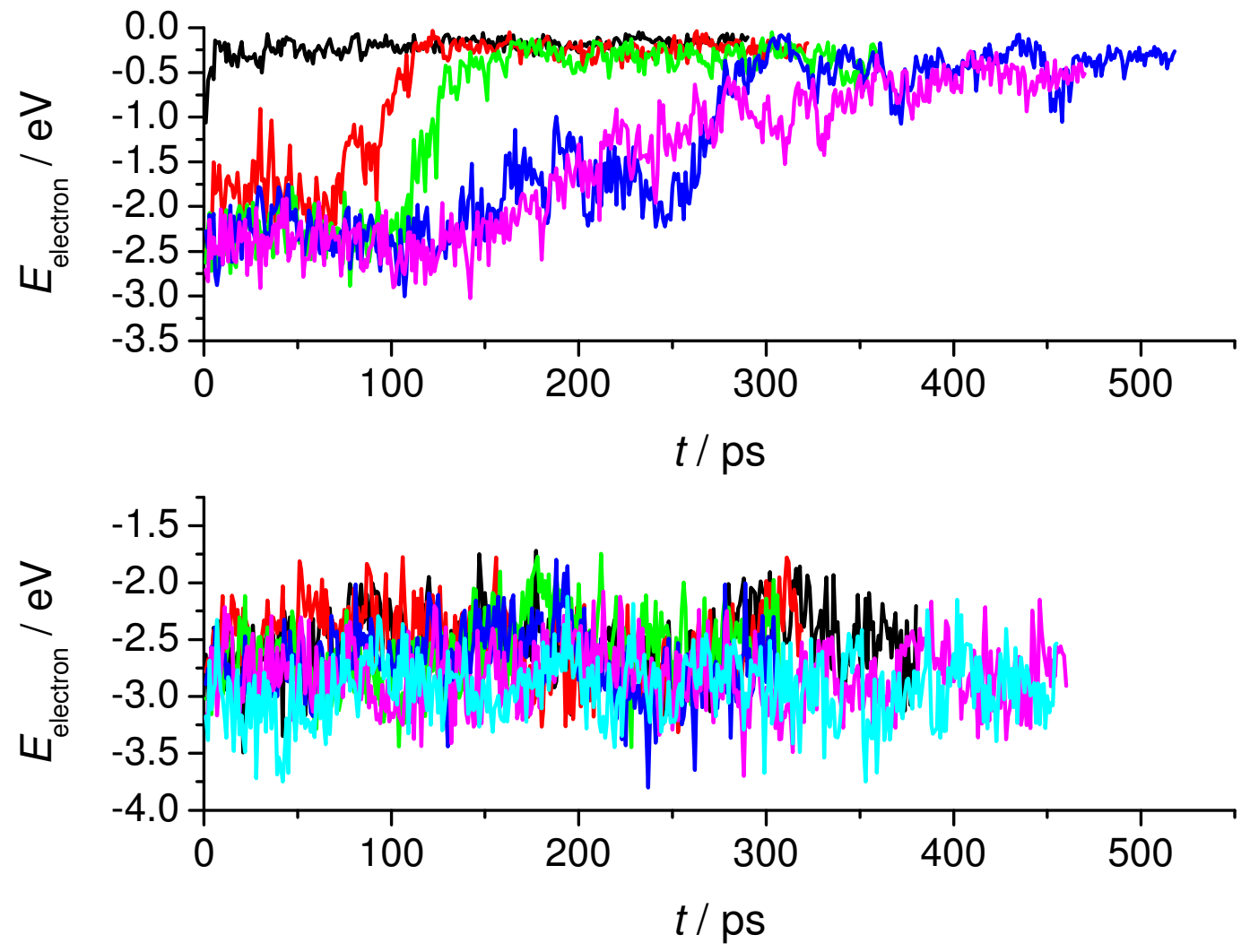
Figure 4. Mones, Rossky and Turi
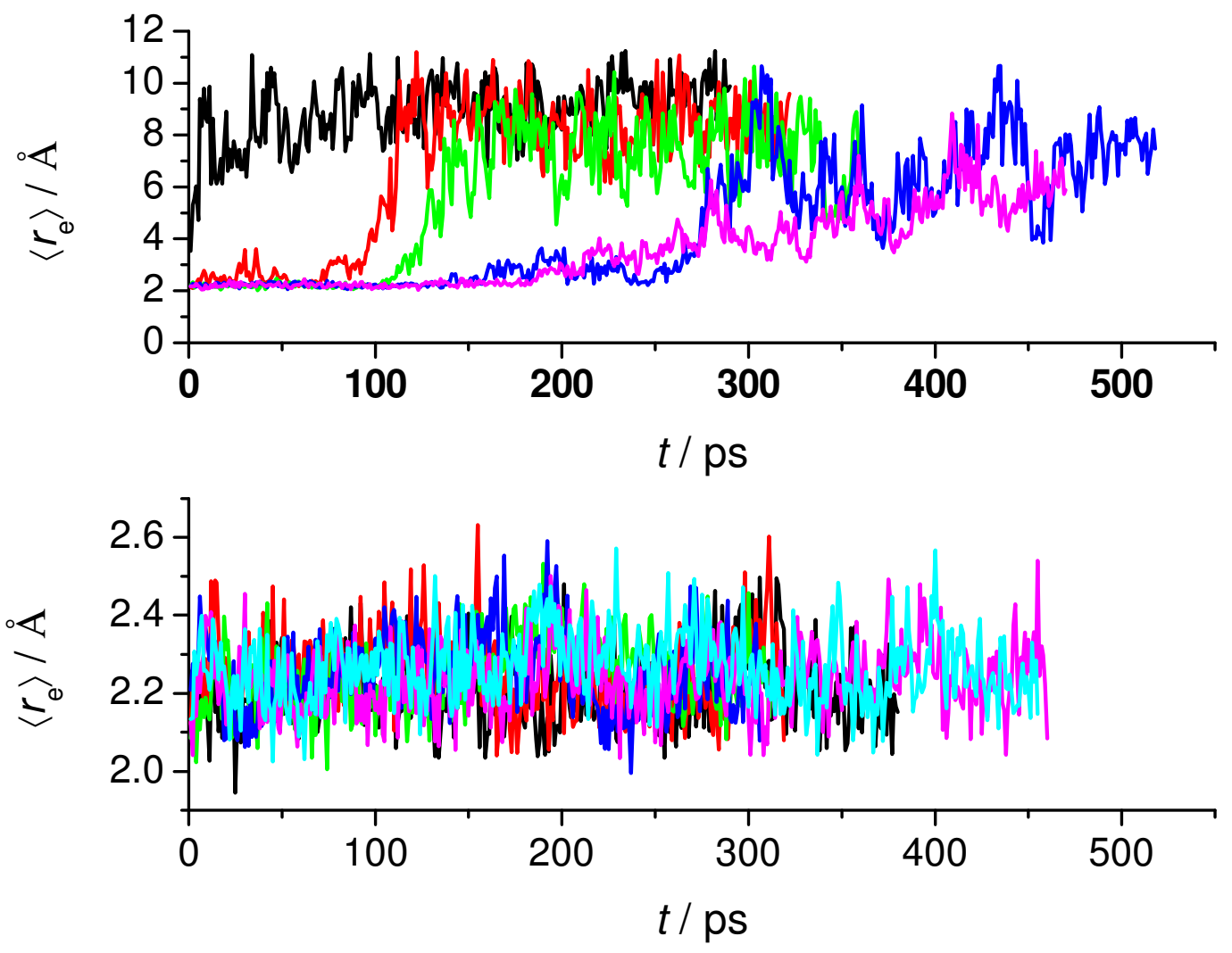
Figure 5. Mones, Rossky and Turi

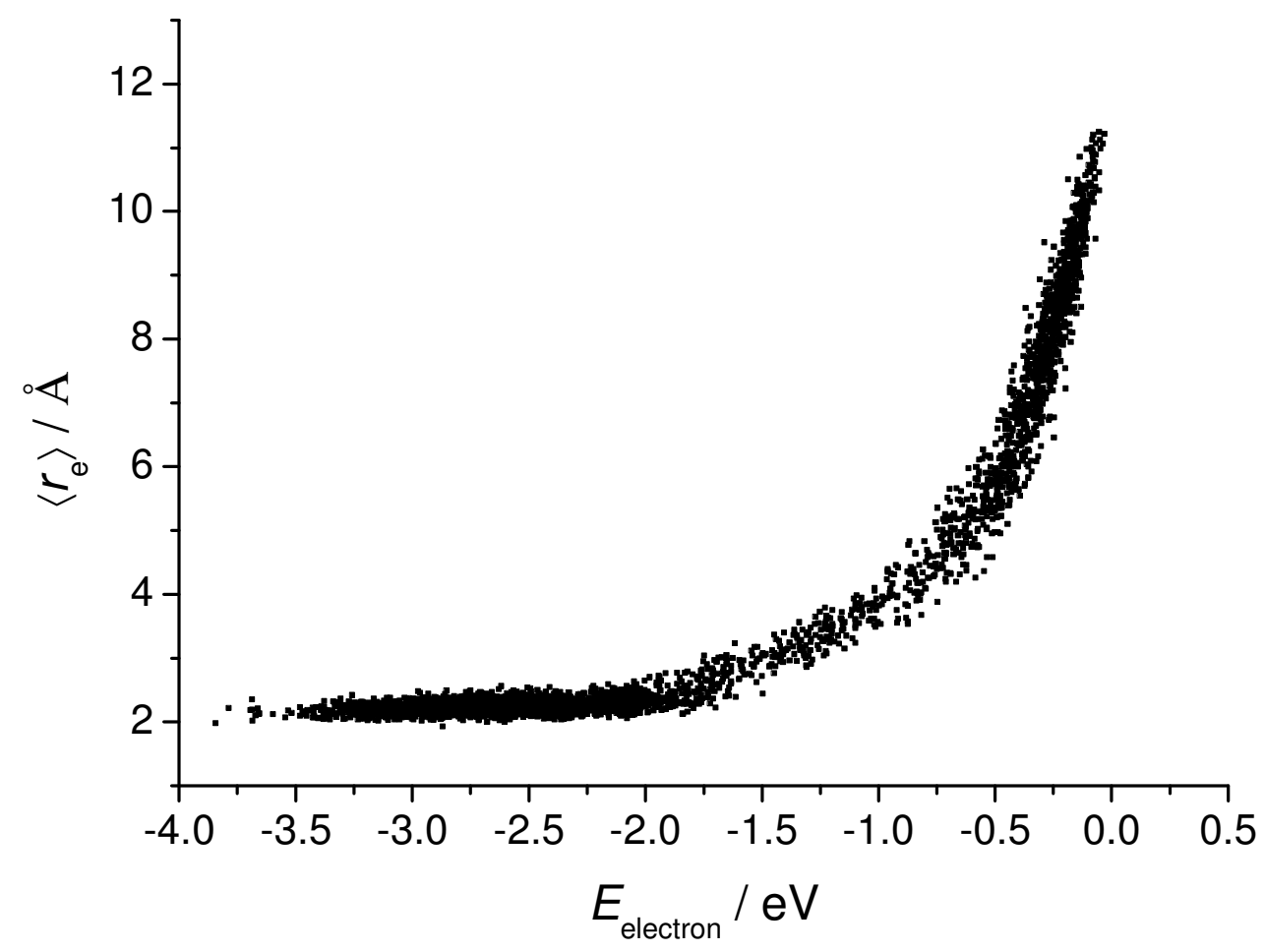


Figure 6. Mones, Rossky and Turi

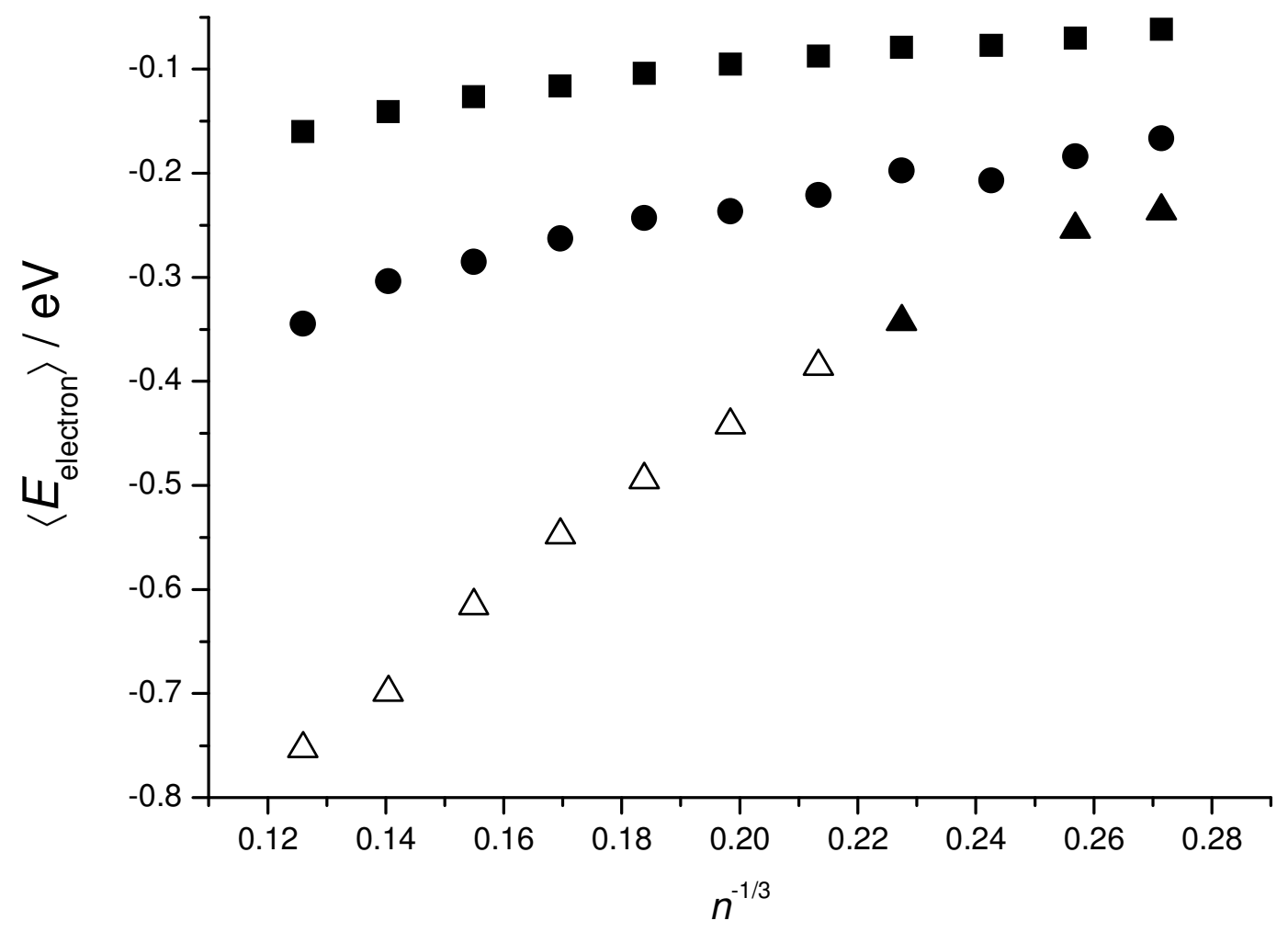


Figure 7. Mones, Rossky and Turi
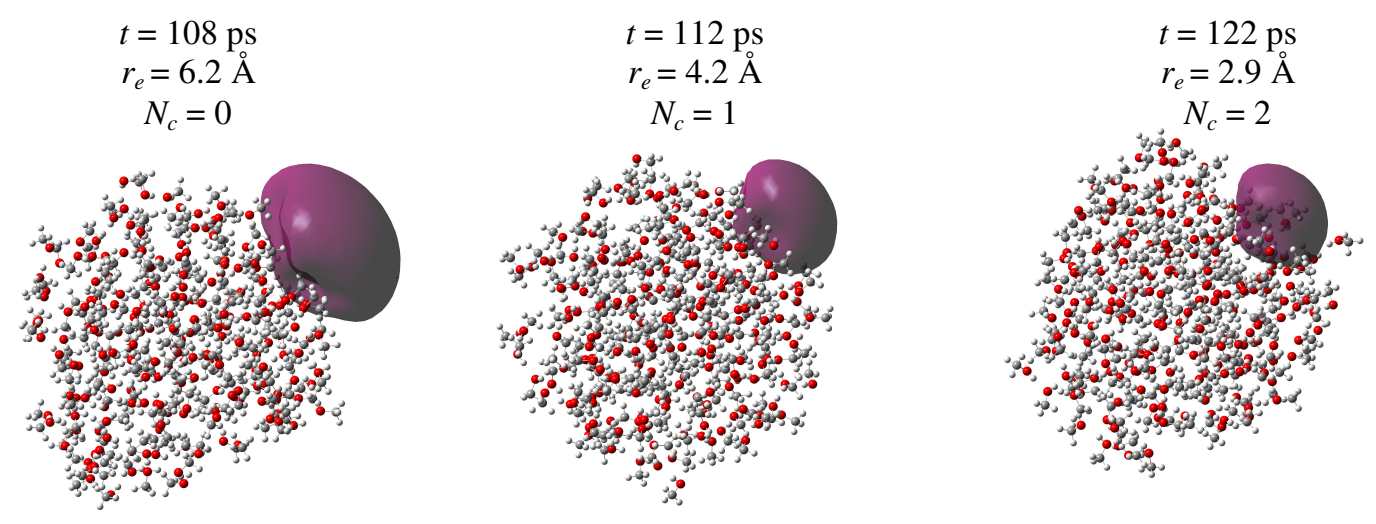

$$
\begin{gathered}
t=131 \mathrm{ps} \\
r_{e}=2.9 \AA \\
N_{c}=3
\end{gathered}
$$

$$
\begin{gathered}
t=146 \mathrm{ps} \\
r_{e}=2.5 \AA \\
N_{c}=4
\end{gathered}
$$

$$
\begin{gathered}
t=153 \mathrm{ps} \\
r_{e}=2.6 \AA \\
N_{c}=4
\end{gathered}
$$
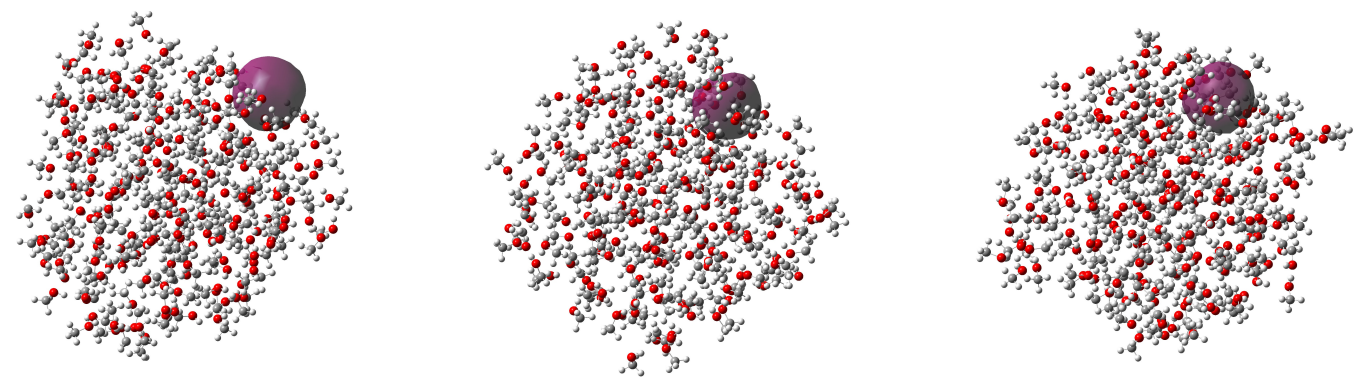

$$
\begin{gathered}
t=158 \mathrm{ps} \\
r_{e}=2.4 \AA \\
N_{c}=4
\end{gathered}
$$

$$
\begin{gathered}
t=163 \mathrm{ps} \\
r_{e}=2.4 \AA \\
N_{c}=4
\end{gathered}
$$

$$
\begin{gathered}
t=173 \mathrm{ps} \\
r_{e}=2.2 \AA \\
N_{c}=5
\end{gathered}
$$
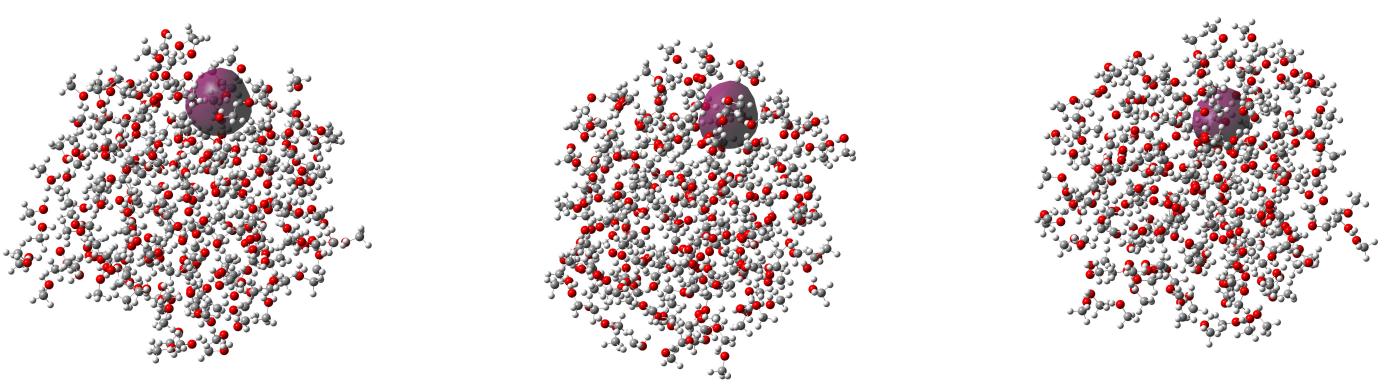
Figure 8. Mones, Rossky and Turi

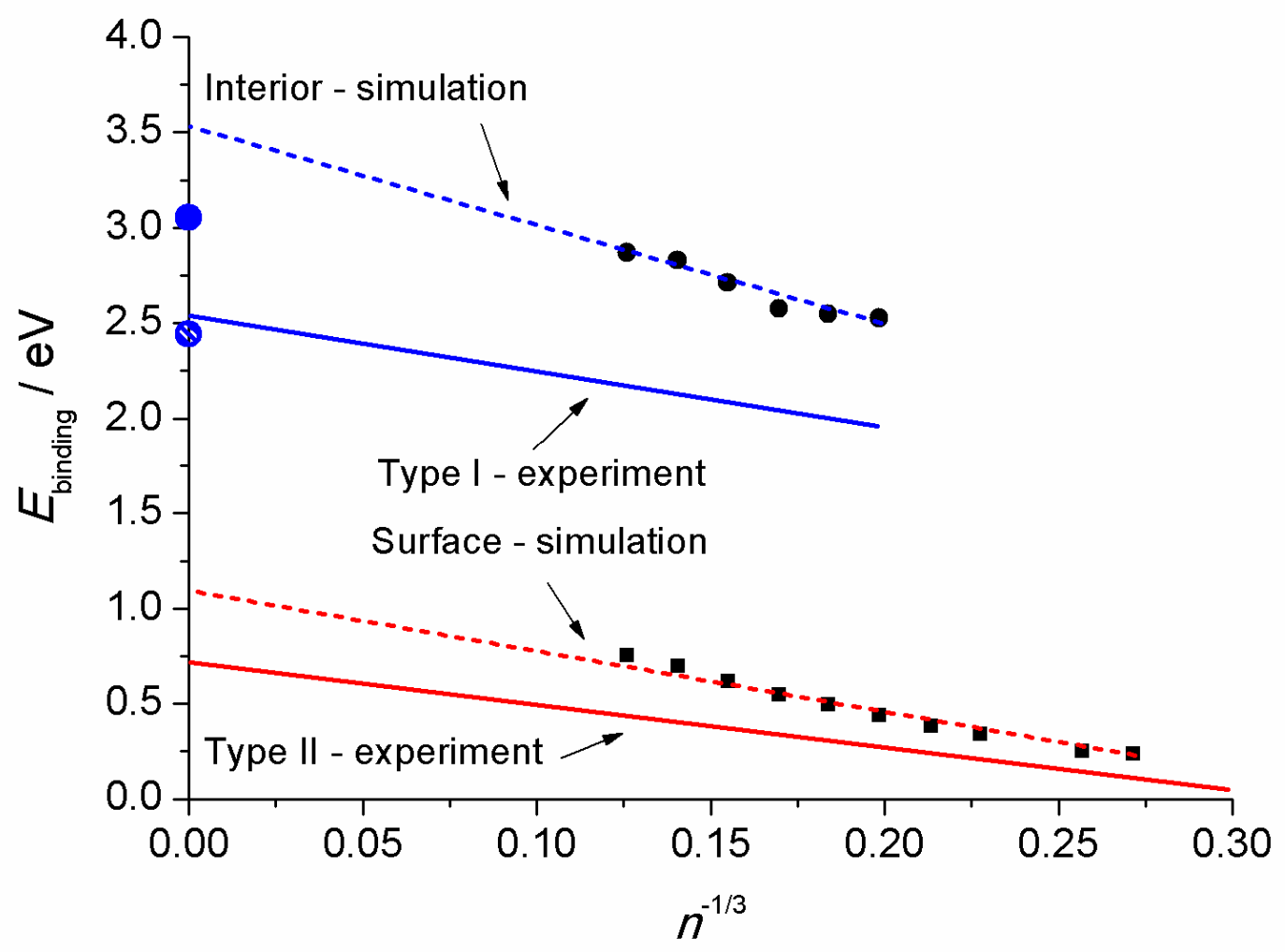


Figure 9. Mones, Rossky and Turi

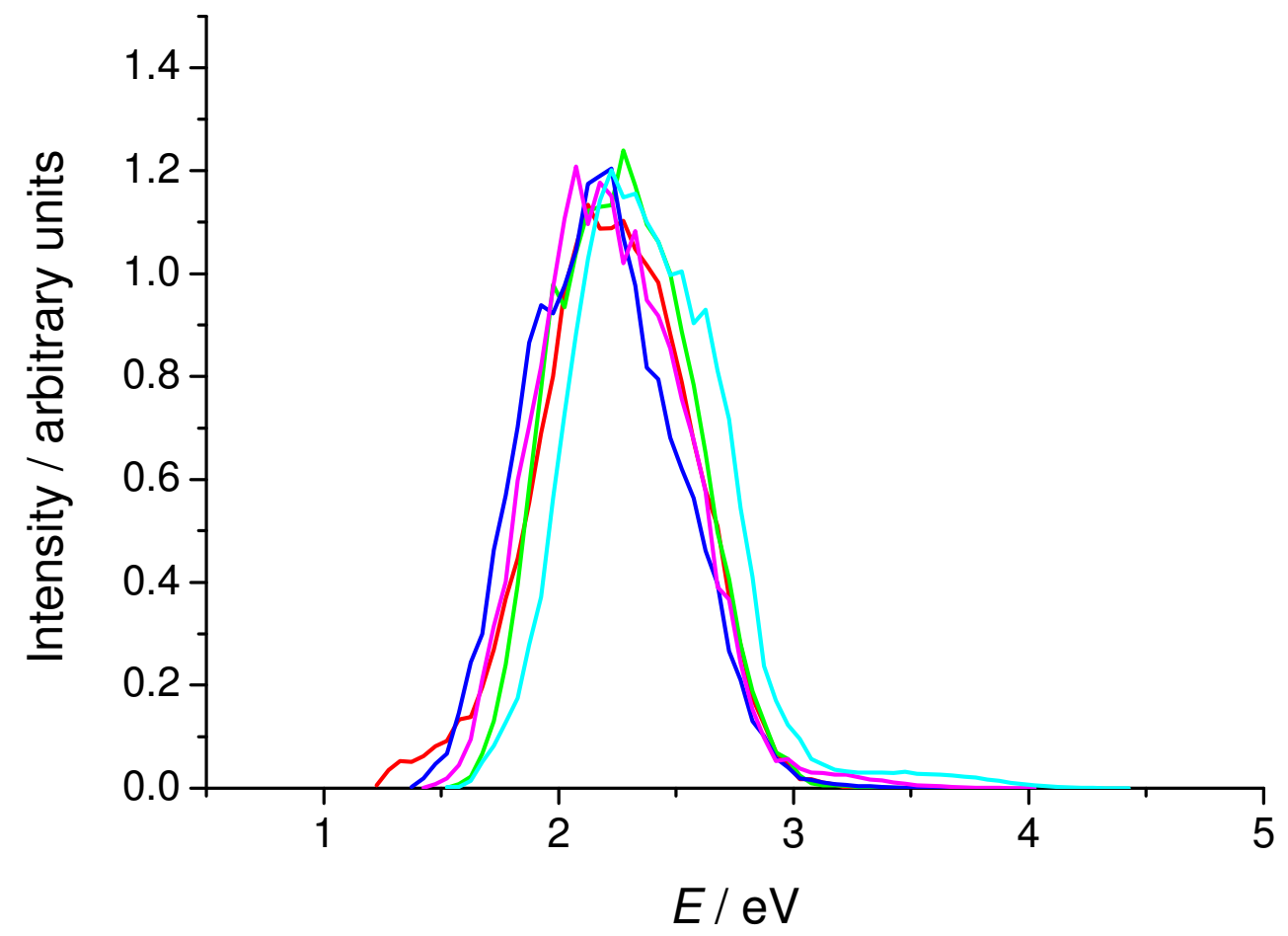




\section{References}

${ }^{1}$ J. H. Baxendale and P. Wardman, J. Chem. Soc., Faraday Trans. 1 69, 584 (1973).

2 a) D. F. Feng and L. Kevan, Chem. Rev. 80, 1 (1980); b) L. Kevan, Radiat. Phys. Chem. 17, 413 (1981); c) L. Kevan, Chem. Phys. Lett. 66, 578 (1979).

${ }^{3}$ F. Y. Jou and G. R. Freeman, Can. J. Chem. 57, 591 (1979); F. Y. Jou and G. R. Freeman, J. Phys. Chem. 81, 909 (1977).

${ }^{4}$ X. Shi, F. H. Long, H. Lu and K. B. Eisenthal, J. Phys. Chem. 99, 6917 (1995).

${ }^{5}$ P. K. Walhout, J. C. Alfano, Y. Kimura, C. Silva, P. Reid and P. F. Barbara, Chem. Phys. Lett. 232, 135 (1995).

${ }^{6}$ C. M. Stuart, M. J. Tauber and R. A Mathies, J. Phys. Chem. A 111, 8390 (2007).

${ }^{7}$ H. Shen, N. Kurahashi, T. Horio, K. Sekiguchi and T. Suzuki, Chem. Lett. 39, 668 (2010).

${ }^{8}$ C. Desfrancois, H. Abdoul-Carime, N. Khefila, J. P. Schermann, V. Brenner and P. Millie, J. Chem. Phys. 102, 4952 (1995).

${ }^{9}$ A. Kammrath, J. R. R. Verlet, G. B. Griffin, and D. M. Neumark, J. Chem. Phys. 125, 171102 (2006).

${ }^{10}$ A. Kammrath, G. B. Griffin, J. R. R. Verlet, R. M. Young, and D. M. Neumark, J. Chem. Phys. 126, 244306 (2007).

${ }^{11}$ R. M. Young, M. A. Yandell and D. M. Neumark J. Chem. Phys. 134, 124311 (2011).

${ }^{12}$ D. H. Paik, I-R. Lee, D.-S. Yang, J. S. Baskin, and A. H. Zewail, Science 306, 672 (2004).

${ }^{13}$ J. R. R. Verlet, A. E. Bragg, A. Kammrath, O. Cheshnovsky, and D. M. Neumark, Science 307, 93 (2005).

${ }^{14}$ L. Ma, K. Majer, F. Chirot and B. v. Issendorff, J. Chem. Phys. 131, 144303 (2009).

${ }^{15}$ L. Turi, W.-S. Sheu, and P. J. Rossky, Science 309, 914 (2005).

${ }^{16}$ Á. Madarász, P. J. Rossky, and L. Turi, J. Phys. Chem. A 114, 2331 (2010).

${ }^{17}$ Z. Kotler, E. Neria and A. Nitzan, Comput. Phys. Commun. 63, 243 (1991). 
${ }^{18}$ F. A. Webster, P. J. Rossky and R. A. Friesner, Comput. Phys. Commun. 63, 494 (1991).

${ }^{19}$ A. Staib and D. Borgis, J. Chem. Phys. 103, 2642 (1995).

${ }^{20}$ L. Turi J. Chem. Phys. 110, 10364 (1999).

${ }^{21}$ J. Zhu and R. I. Cukier, J. Chem. Phys. 98, 5679 (1993).

${ }^{22}$ L. Turi, A. Mosyak, and P. J. Rossky, J. Chem. Phys. 107, 1970 (1997).

${ }^{23}$ A. Mosyak, P. J. Rossky, and L. Turi, Chem. Phys. Lett. 282, 239 (1998).

${ }^{24}$ A. Mosyak, O. V. Prezhdo, and P. J. Rossky, J. Mol. Struct. 485-486, 545 (1999).

${ }^{25}$ P. Mináry, L. Turi, and P. J. Rossky, J. Chem. Phys. 110, 10953 (1999).

${ }^{26}$ L. Turi, P. Mináry, and P. J. Rossky, Chem. Phys. Lett. 316, 456 (2000).

${ }^{27}$ L. Turi and P. J. Rossky, J. Chem. Phys. 120, 3688 (2004).

${ }^{28}$ L. Mones and L. Turi, J. Chem. Phys. 132, 154507 (2010).

${ }^{29}$ L. Mones, P. J. Rossky and L. Turi, J. Chem. Phys. 133, 144510 (2010).

${ }^{30}$ L. Turi, Á. Madarász, and P.J. Rossky, J. Chem. Phys. 125, 014308 (2006).

${ }^{31}$ U. Buck and F. Huisken, Chem. Rev. (Washington, D.C.) 100, 3863 (2000).

${ }^{32}$ M. Knapp, O. Echt, D. Kreisle and E. Recknagel, J. Phys. Chem. 91, 2601 (1987).

${ }^{33}$ F. Zappa, S. Denifl, I. Mähr, A. Bacher, O. Echt, T. D. Märk, and P. Scheier, J. Am. Chem. Soc, 130, 5573 (2008).

34 Á. Madarász, P. J. Rossky, and L. Turi, J. Chem. Phys. 130, 124319 (2009).

${ }^{35}$ W. L. Jorgensen, D. S. Maxwell, and J. Tirado-Rives, J. Am. Chem. Soc. 118, 11225 (1996).

${ }^{36}$ L. Turi, M.-P. Gaigeot, N. Levy and D. Borgis, J. Chem. Phys. 114, 7054 (2001).

${ }^{37}$ L. Turi, and D. Borgis, J. Chem. Phys. 117, 6186 (2002).

${ }^{38}$ Á. Madarász, P. J. Rossky, and L. Turi, J. Chem. Phys. 126, 234707 (2007).

${ }^{39}$ T. Frigato, J. VandeVondele, B. Schmidt, C. Schütte, and P. Jungwirth, J. Phys. Chem. A 112, 6125 (2008). 
${ }^{40}$ O. Marsalek, F. Uhlig, T. Frigato, B. Schmidt and P. Jungwirth, Phys. Rev. Lett. 105, 043002 (2010).

${ }^{41}$ To check the convergence of surface state calculations we also performed short simulations with $64 \times 64 \times 64$ grid points in a box of $50 \AA$ A length for smaller clusters. The convergence of the interior state calculations was checked for Ref. 28.

${ }^{42}$ Of the surface-state initiated trajectories, in the $n=70$ case, the electron became too diffuse to be physical. For this reason, we left this cluster out of the analysis.

${ }^{43}$ W. C. Swope, H. C. Andersen, P. H. Berens, and K. R. Wilson, J. Chem. Phys. 76, 637 (1982).

${ }^{44}$ The $n=269$ case was an exception, where only a 15 ps long trajectory portion with stable surface state behavior was found.

${ }^{45}$ G. Makov, and A. Nitzan, J. Phys. Chem. 98, 3459 (1994).

${ }^{46}$ L. D. Jacobson and J. M. Herbert, J. Am. Chem. Soc. 132, 10000 (2010). 